\title{
Circulating tumour cell clusters: Insights into tumour dissemination and metastasis
}

Sayuri Herath , Sajad Razavi Bazaz , James Monkman , Majid Ebrahimi Warkiani, Derek Richard, Ken O'Byrne \& Arutha Kulasinghe

To cite this article: Sayuri Herath, Sajad Razavi Bazaz, James Monkman, Majid Ebrahimi Warkiani , Derek Richard, Ken O'Byrne \& Arutha Kulasinghe (2020): Circulating tumour cell clusters: Insights into tumour dissemination and metastasis, Expert Review of Molecular Diagnostics, DOI: $10.1080 / 14737159.2020 .1846523$

To link to this article: https://doi.org/10.1080/14737159.2020.1846523

Accepted author version posted online: 03

Nov 2020.

Submit your article to this journal $\sqsubset$

山 Article views: 23

Q View related articles $\longleftarrow$

View Crossmark data $־$ 
Publisher: Taylor \& Francis \& Informa UK Limited, trading as Taylor \& Francis Group

Journal: Expert Review of Molecular Diagnostics

DOI: $10.1080 / 14737159.2020 .1846523$

\title{
Circulating tumour cell clusters: Insights into tumour dissemination and metastasis
}

\author{
Sayuri Herath ${ }^{1}$, Sajad Razavi Bazaz ${ }^{2}$, James Monkman ${ }^{3,4,5}$, Majid Ebrahimi Warkiani ${ }^{2,6}$, Derek \\ Richard $^{3,4,5}$, Ken O’Byrne ${ }^{3,4,5,7}$, Arutha Kulasinghe ${ }^{3,4,5, *}$ \\ 1 Department of Medical Laboratory Sciences, Faculty of Health Sciences, The Open University of \\ Sri Lanka \\ 2 School of Biomedical Engineering, University of Technology Sydney, NSW, Australia. \\ 3 The School of Biomedical Sciences, Institute of Health and Biomedical Innovation, \\ Queensland University of Technology, Brisbane, QLD, Australia. \\ 4 Translational Research Institute, Woolloongabba, QLD, Australia. \\ 5 Cancer and Ageing Research Program, Australia \\ 6 Institute of Molecular Medicine, Sechenov First Moscow State University, Moscow 119991, Russia \\ 7 Princess Alexandra Hospital, Woolloongabba, QLD, Australia. \\ *Corresponding Author: \\ Arutha Kulasinghe \\ Address: The School of Biomedical Sciences, Institute of Health and Biomedical Innovation at the Translational \\ Research Institute, Queensland University of Technology, 37 Kent Street, Woolloongabba, Queensland 4102, \\ Australia. Email: arutha.kulasinghe@qut.edu.au
}




\section{Abstract}

\section{Introduction:}

Metastasis results in more than $90 \%$ of cancer related deaths globally. The process is thought to be facilitated by metastatic precursor cells, commonly termed circulating tumour cells (CTCs). CTCs can exist as single cells or cell clusters and travel through the lymphovasculature to distant organs where they can form overt metastasis.

\section{Areas covered:}

Studies have highlighted that CTC clusters, which may be homotypic or heterotypic in composition, have a higher metastatic potential compared to single CTCs. The characterisation of CTC clusters is becoming important as heterotypic clusters can provide a mechanism for immune evasion. This review summarises the latest advances in CTC cluster mediated metastasis and clinical significance.

\section{Expert Opinion:}

Comprehensive characterisation of CTC clusters is needed to understand the cell types and interactions within clusters, in order to identify ways in which to reduce CTC cluster mediated metastasis. The role of CTC clusters in prognosticating disease progression needs to be determined by documenting CTC clusters from the time of diagnosis over the course of therapy.

Keywords: Circulating tumour cells, CTC clusters, homotypic, heterotypic, metastasis. 


\section{Article highlights}

- CTC clusters are more efficient at seeding metastasis than single CTCs

- CTC clusters have a higher metastatic capacity than single CTCs

- CTC clusters can be composed of only tumour cells (homotypic) or a milleiu of stromal, immune and tumour cells (heterotypic)

- Heterotypic CTC clusters are able to evade the immune surveillance mechanisms

- CTC clusters are rare cells in the blood and advanced isolation and enrichment strategies are required for their capture

- Advances in microfluidic technologies has enabled the capture of CTC clusters 


\section{Introduction}

The seed and soil theory, hypothesized by Stephen Paget, was pivotal for the understanding of the metastatic process. It was proposed that metastatic dissemination is not a random process and it is determined by the dynamic interplay between tumour cells with metastatic potential (seeds) and a compatible host microenvironment (soil) [1]. The infiltration of a specific organ by the metastatic tumour cells is dependent on the cellular signaling from the tumour stroma, the composition of CTCs, the structure of the target organ microcirculation and cancer-cellautonomous functions [2]. CTCs were first described by an Australian physician, Thomas Ashworth in 1869, where he made the observation that cells similar to that of the primary tumour were found in the blood of a patient with metastatic disease. Since these findings, the field has rapidly emerged, powered in part by advancements in CTC enrichment technologies. However, there remains only 1 FDA approved platform - the CellSearch (Menarini Silicon Biosystems). This platform has demonstrated clinical associations of CTC enumeration with progression free survival (PFS) and overall survival (OS) in a number of solid tumours.

CTCs can exist as single cells or clusters. Aggregation of two or more CTCs through intracellular junctions are known as CTC Clusters $[3,4,5,6]$. From a series of mouse model experiments, Aceto et al. (2014) revealed that CTC clusters are derived from primary tumour cells, denoting an oligoclonal origin [7]. A recent study in breast cancer indicated that plakoglobin, a major cytoplasmic component of both desmosomes and adherens junctions, works as a key factor for tumour cell clustering [8]. The study emphasized that downregulation of plakoglobin leads to a reduction of CTC cluster formation and lowering of the metastatic capacity [7]. More recently, two studies discovered that tight junction proteins claudin 3 and claudin 4 play a significant role in cluster formation [7,9]. The studies revealed that intracellular junctions have a more profound effect than the cell to cell interactions for the formation of metastasis. It has also been shown that DNA methylation dynamics significantly impact on CTC cluster formation which enhances stemness and metastatic seeding ability. Aggregation of CTCs leads to hypomethylation of binding sites for stemness and proliferation-associated transcription factor and the disruption of multicellular structures in CTC clusters causes re-methylation of 
transcription factor binding sites and suppression of metastatic dissemination [9]. A summary of CTC clusters has been detailed in Table 1 .

Metastasis is thought to be facilitated by a series of interrelated events. However, it is still not fully understood how CTCs intravasate into the lymphovasculature [10]. It has been speculated that a proportion of tumour cells are released into the blood or lymphatic circulation by actively traversing the endothelial cell barrier while some cells shed passively into the circulation as a result of lack of tumour vasculature (Figure 1) [10]. A major emphasis has been placed on the low adhesiveness of CTCs and noted that decrease adhesiveness of CTCs also triggers the metastatic potentiality [11] as changing of cellular stiffness and contractility facilitate the immediate transversion via loosen endothelium [12]. The extravasation step of the metastasis process is thought to be mediated by a variety of endoproteases secreted by CTCs, namely matrix metalloproteases (MMPs), cysteine proteases and desintegrins [13].

It was believed that CTC clusters were entrapped at the small lumen of capillaries and that would likely be the point of extravasation. However, in silico, in vivo and in vitro studies proved that CTC clusters were able to traverse capillaries by unfolding into single-file formats with greater efficiency (Figure 2) [14]. Unfolding of CTC clusters into a single geometric chains largely reduces the overall hydrodynamic resistance and is thought to facilitate metastatic dissemination [14]. Finally, adhesion and development of metastatic deposits take place at the distant sites of the body [15]. 


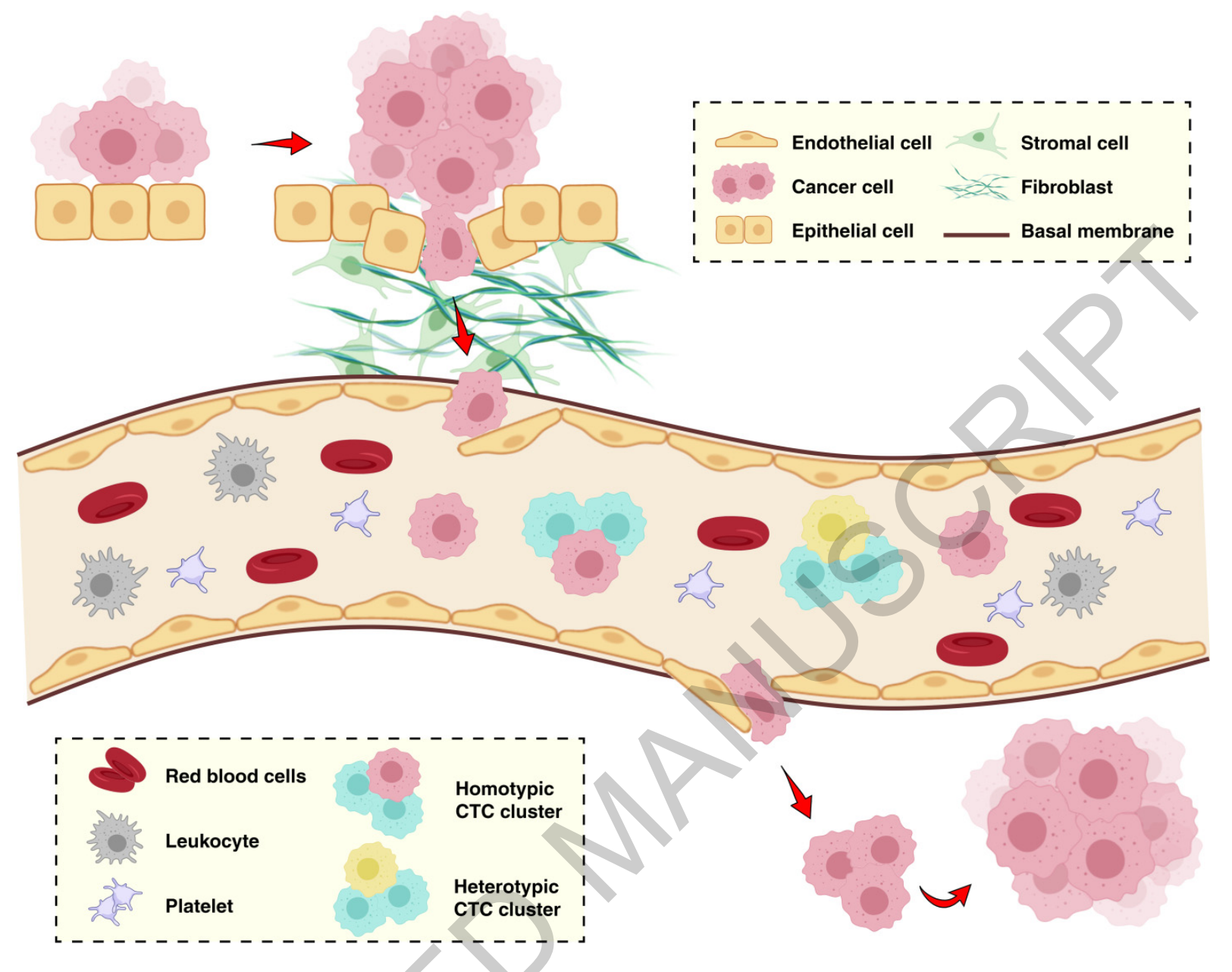

Figure 1. CTC clusters in circulation.

The metastatic cascade initiates with the detaching and movement of individual tumour cells or tumour cell aggregates (homotypic/heterotypic) from the primary or secondary tumour foci into the lymphovasculature. In circulation, single CTCs and CTC clusters evade the body's immune system and stressors in the blood. At favorable sites, the CTCs extravasate by traversing the endothelial cell barrier to form micro/macrometastasis. 


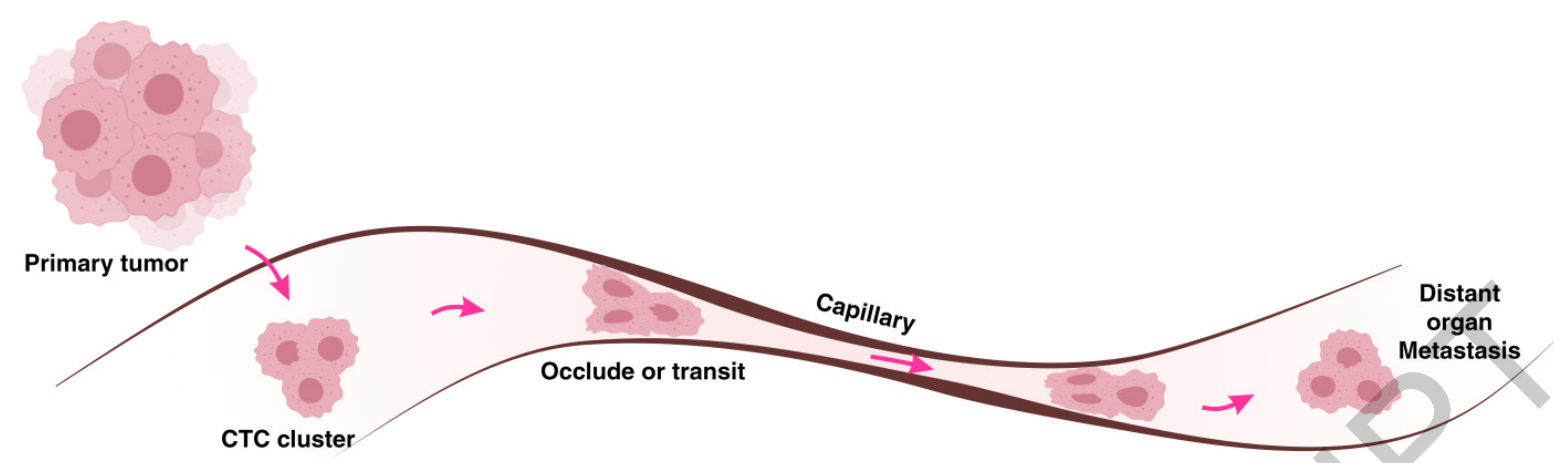

Figure 2. CTC clusters retain their morphology whilst traversing narrow capillaries.

It was assumed that CTC clusters were too large to traverse narrow capillaries to reach distance organs and were thought to metastasize at the site where the cluster got trapped in the capillary. Recent data has revealed that at narrow capillaries, CTC clusters unfold into elongated single-file chains and transverse narrow vessels. When the vessels enlarges, the CTC clusters reforms.

\section{Epithelial-mesenchymal plasticity of CTCs}

Epithelial-mesenchymal transition (EMT) is an intricate biological process that results in the gradual suppression of epithelial features of the tumour cells and acquires mesenchymal features in order to obtain higher level of plasticity, migratory and invasive metastatic characteristics as well as immune evasion [16]. The transition of an epithelial cell into a mesenchymal cell requires changes in cellular morphology, cellular architecture, adhesion molecules and migration capacity. It has been speculated that during EMT, downregulation of epithelial biomarkers such as cytokeratins, E-cadherin, desmoplakin, occludin and Epithelial Cell Adhesion Molecule (EpCAM) takes place, while the upregulation of mesenchymal markers such as fibronectin, vimentin, N-cadherin and transcription factors such as Snail1 (Snail), Snail2 (Slug), Twist, EF1/ZEB1, SIP1/ZEB2, E47 take place $[17,18]$. $\beta$-Catenin displays a dual function in the EMT process. When binding with cadherin complexes in adherens junctions, it boosts cell to cell adhesion and works as a transcriptional coactivator in the nucleus [19]. Epidermal Growth Factor (EGF) has been found as an EMT inducer which is caused by downregulating E-cadherin production and upregulating vimentin production [20]. Another study has been revealed that transforming growth factor- $\beta$ (TGF- $\beta$ ) plays a major role in EMT regulation. It induces EMT via activation of HEY1 gene which is a hairy/enhancer-of-split family of transcriptional repressors 
[21]. Further, several studies documented that integrin- $\alpha v \beta 6$ as one of the key player in mesenchymal cells which enhance migration, survival and inactivation of apoptosis of tumour cells $[22,23]$. Furthermore, during this transition process, proteins such as $\beta$-catenin, Smad- $2 / 3$, NF- $\kappa \beta$, Snail, Slug and Twist are accumulated within the nuclei and cells acquire higher migration, three-dimensional invasion, scattering capacity, changing the shape as a more elongated pattern and resistance to anoikis [17]. Moreover, MET receptor tyrosine kinase, a cell surface receptor has been identified as a stimulator for the scattering of epithelial cells in the process of EMT.

EMT causes resistance to anoikis, chemotherapy, the mechanical shearing forces and senescence which are crucial for CTC survival and dissemination $[24,25,26]$. CTCs or CTC clusters exhibit epithelial to mesenchymal properties partially or totally [27]. In the established metastatic deposits, tumour cells primarily show epithelial features hence, MET is essential to re-acquire their proliferative ability [28]. The histological resemblance between secondary metastatic deposits and the primary tumour suggests that EMT mediated metastatic development is thought to be followed by a reverse MET to colonize secondary sites [17].

\section{Composition of CTC clusters}

Whilst much is known about the primary and metastatic tissues, there is limited data available for CTCs. Therefore, robust characterisation of CTCs is needed in order to improve the effectiveness of cancer management and develop anti-metastatic treatment strategies [9, 11]. CTC clusters can be broadly categorized into two groups; homotypic and heterotypic CTC clusters. When CTC clusters only contain tumour cells, they are designated as homotypic CTC clusters where they are typically made up of between 1-30 CTCs [7, 29]. Heterotypic CTC clusters are cellular aggregates which are made of cancer cells as well as non-cancerous stromal or immune cells. Non-cancerous cells include white blood cells, fibroblasts, endothelial cells and platelets [11] and the type of non-cancerous cells present depends on the tumour type and its abundance of particular immune or stromal cells (Figure 3) [30, 31]. These non-cancerous cells are thought to be advantageous for the survival of CTC clusters by promoting proliferation, and maintaining resistance against host immune responses [32] [31, 33]. 


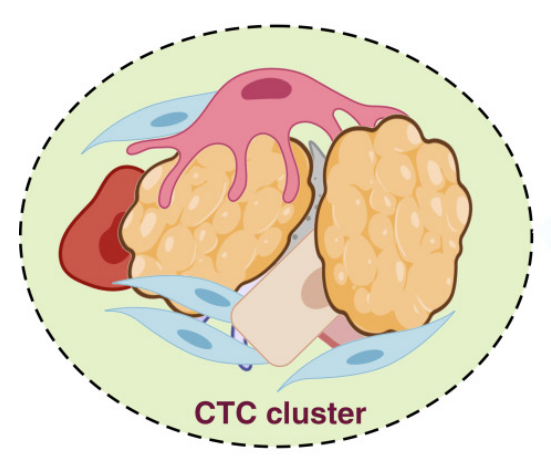

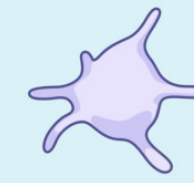

Platelet

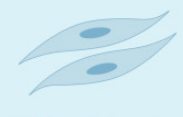

Fibroblasts

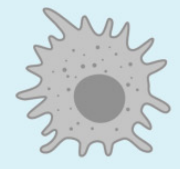

Leukocytes

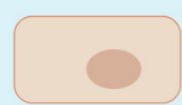

Endothelial cells
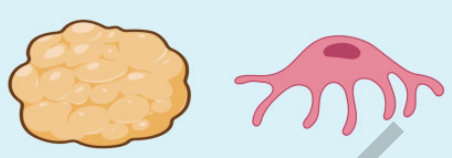

Tumor cells

Pericytes

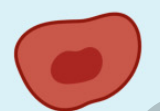

Plakoglobin + Tumor cells

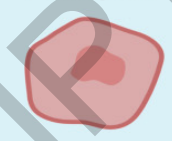

$\mathrm{k} 14+$

Tumor cells

Figure 3. Cellular composition of CTC clusters.

CTC clusters are made of cancer cells (homotypic) as well as non-cancerous stromal or immune cells bind through intracellular junctions (heterotypic). Non-cancerous cells include leucocytes, fibroblasts, endothelial cells and platelets. It is thought that heterotypic clusters have an increased survival advantage and immune evasion strategy by having immune cells integrated into the cluster.

In the vast majority of cases, heterotypic CTC clusters comprise of the cells from the various stages of myeloid lineage. For instance, tumour-infiltrating neutrophils depart from the primary tumour with the cancer cells and released to the vasculature in the form of CTC-neutrophil clusters [31]. Formation of CTC-neutrophil clusters is mediated by a cytokine-receptor crosslink which involves Interleukin $1 \mathrm{~b}$ (IL-1b) and Interleukin 6 (IL-6). Thus, reduction of IL-1b and IL6 resulting in a lowering the formation of CTC-neutrophil clusters [31]. The study has pointed out that CTC-neutrophil clusters are bound together through vascular cell adhesion molecule-1 (VCAM-1) dependent intercellular junctions and downregulation of VCAM-1 have been caused to prevent the formation of CTC-neutrophil clusters. The study has further revealed that CTCneutrophil clusters accelerate the metastatic seeding potential by assisting DNA replication and cell cycle progression in the circulation and eventually lead to poor prognosis [31].

Recent studies have highlighted that CTC clusters have 23-50 times higher metastatic potential compared to single CTCs and immune evasion mechanism to avoid surveillance from the 
immune system [7]. The greater metastatic potential of CTC clusters may be due to their multicellular features which increases the molecular heterogenicity [14, 34]. Furthermore, expression of the epithelial cytoskeletal protein keratin 14 (K14) in CTC clusters, has been identified as a key player in achieving metastatic dissemination [34]. Therefore, studies have suggested that the presence of CTC clusters associated with adverse clinical outcomes and poor prognosis $[35,36,37]$. Due to the presence of multiple cancer cells together, CTC Clusters have a better survival rate than single CTCs in circulation. However, their presence in the peripheral blood or lymphatic circulation is extremely rare and it constitutes only 2-3\% of all CTCs. By applying in vivo flow cytometric techniques to mouse models, a study has been determined the half-life of CTC clusters as approximately 6 to 10 minutes while it is 25 to 30 minutes in single CTCs. In addition, CTC clusters are thought to be removed from the circulation faster than single CTCs, with a greater likelihood to metastasize than single CTCs [7].

\section{Immune evasion of CTCs}

CTCs have distinct characteristics such as EMT, dormancy, survival in the bloodstream, immune evasion, chemo-resistance and ability to escape from the anti-cancer immune responses [38, 39]. The ability of CTCs to undergo EMT enable the cancer cells to escape the primary tumour site and intravasate into the circulation [40]. Kim et al., (2012) showed that CTCs are resistant to anoikis and thereby are suited to survive in the vasculature [41]. However, a study conducted by Luzzi et al. (1998) showed that the overwhelming majority of CTCs are subjected to die due to anoikis and only $2.5 \%$ of CTCs appeared to form micrometastases while macrometastases developed from $0.01 \%$ of CTCs [42]. Studies showed that expression of abnormal genes such as survivin, epidermal growth factor receptor and immunosuppressive molecules may cause inhibition of apoptosis and thereby survival of CTCs. Survivin is a main apoptosis protein inhibitor that evades the immune system by blocking the cytotoxic Natural Killer (NK) cells. Furthermore, it impedes apoptotic proteases such as caspase- 8 and caspase-6 [43]. Moreover, epidermal growth factor receptor also plays a major role in tumour cell proliferation, inhibition of apoptosis and angiogenesis. Upregulation of immunosuppressive molecules also fosters the immune evasion of CTCs by resisting tumour antigen-specific T lymphocytes and NK cells [43]. 
Recent studies highlighted that programmed cell death-1 (PD-1) receptor is a key component in the cancer-immune evasion mechanism [44, 45]. PD-1 is a negative immune-regulatory checkpoint, which is expressed on activated T cells [43]. In contrast, programmed cell death ligand 1 (PD-L1) is present on the cell surface of antigen-presenting cells such as macrophages or dendritic cells $[46,47]$. PD-L1 plays a central role in adaptive cellular immunity, controlling the T-cell activation and differentiation [43]. When PD-L1 binds to its receptor, PD-1, a strong inhibitory signal is conveyed into the $\mathrm{T}$ lymphocyte, which then influences on the reduction of cytokine production and suppression of T-cell proliferation [46, 47]. A recent study has documented that PD-L1 is frequently expressed on CTCs [48]. Studies have shown that CTC clusters have higher relative PD-L1 expression compared to known models [45]. Furthermore, overexpression of PD-L1 or PD-L2 by tumour cells activates the PD-1 checkpoint pathway, by engaging with PD-1 receptors [49]. This interaction results an immune evasion strategy by CTCs $[48,50]$. Moreover, the interaction between PD-L1 with its receptor influences apoptosis of activated T-cells [51]. Therefore, the detection of CTCs with PD-L1 in circulation indicates the attenuation of immune defense mechanism and progression of metastasis which ultimately leads to a poorer patient prognosis [48].

The expression of the human leukocyte antigen-G (HLA-G) in various malignancies has been increasingly observed and strongly linked with tumour immune invasion and metastasis. This is accompanied by the cytolysis of immune cells, downregulating the cytokine production, apoptosis of immune cells and stimulating the generation of regulatory $\mathrm{T}$ cells [52]. In addition to the above protective mechanisms, several other cell types in tumour microenvironment enhance the survival of CTC clusters. For instance, platelets facilitate CTCs existence by protecting them from NK cells and shear stresses [53]. CTCs couple with the reactive platelets and acquire major histocompatibility complex (MHC) whereby they imitate the characteristics of the host cells in order to escape host immune responses [54]. Furthermore, platelets provide physical shielding to CTC clusters and escape from immune mechanisms [55]. Despite the protective role of the immune system, some immune cells trigger CTC survival by promoting the development of an immune resistant microenvironment and maintaining resistance to anoikis and chemotherapy [56]. Moreover, CTC clusters undertake reversible metabolic changes that increase their potential to withstand oxidative stress [57, 58]. 


\section{CTC cluster enrichment and detection technologies}

CTCs are extremely rare, even in patients with metastatic cancer (approximately one cancer cell among a billion normal blood cells) and their isolation is greatly subjected to technological constraints $[59,60]$. However, with improvements in isolation methodologies, the capture of these rare cells is becoming increasingly more achievable. The current CTC enrichment technologies use biological or physical properties of the CTCs for isolation. The CellSearch (Menarini Silicon Biosystems) platform remains the only FDA-approved CTC isolation platform and pre-selects on EpCAM, a transmembrane glycoprotein involved in cell to cell adhesion [61]. However, this platform has a number of limitations with the sensitivity of the method as it potentially does not capture mesenchymally shifted/stem-like CTCs and CTC clusters with low or absent EpCAM expression $[62,63]$.

To overcome these limitations, label-free CTC isolation technologies have been developed which capture CTCs based on size, density and deformability [59]. However, significant drawbacks of label-free technologies are also exhibited and the utility is limited due to throughput [64]. To tackle these shortcomings, recent advances in CTC and CTC cluster capture have been optimized using microfluidic technologies such as the straight, spiral and labyrinth microfluidic platforms (Figure 4) [32, 62, 64]. This method uses the physical properties of CTCs such as size and deformability changes to differentiates CTCs from hematopoietic cells $[65,66]$. The microfluidic technology utilizes electrophoresis, hydrodynamic and cross-flow filtration, micropore and micropost trapping, deterministic lateral displacement and inertial focusing systems to capture CTCs $[67,68,69]$. This has the unique features of efficient cell sorting without need for purification, high system throughputs, a requirement of low sample volume and ability to analyze functions of CTCs in vitro [27, 62, 64, 70, 71]. More importantly, a number of these technologies support processing using undiluted whole blood [62]. However, microfluidic technologies do have limitations such the inability to capture small CTCs $(<14 \mu \mathrm{m})$ and the presence of contaminating leukocytes in CTC channels [72]. To minimize the effects associated with purity of CTCs, recent studies has been established using a multi-flow microfluidic system for capturing CTC populations with high purity [71]. To date, there are limited studies documenting the presence of CTC clusters. One of the limiting factors has been the low numbers of CTC 
clusters per volume of normal blood cells in circulation [32]. Moreover, CTC clusters tend to be dissociated during the blood sample collection and processing [32]. To Address the above, recently several platforms have been developed, namely, the Cluster-Chip, DLD-Chip, photoacoustic technique and antibody-functionalized 3D scaffold gelatin-microchip in order to detect CTC clusters alone [32].

A)

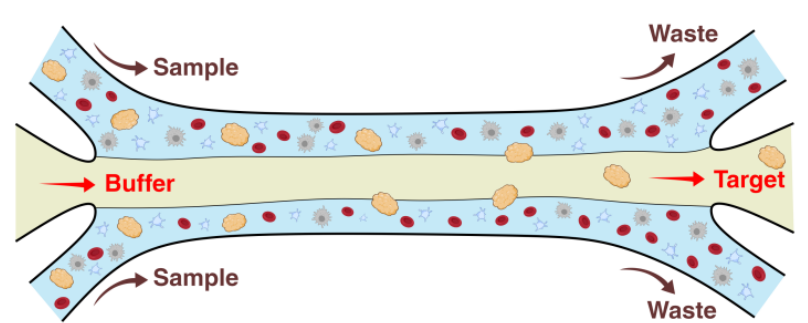

C)
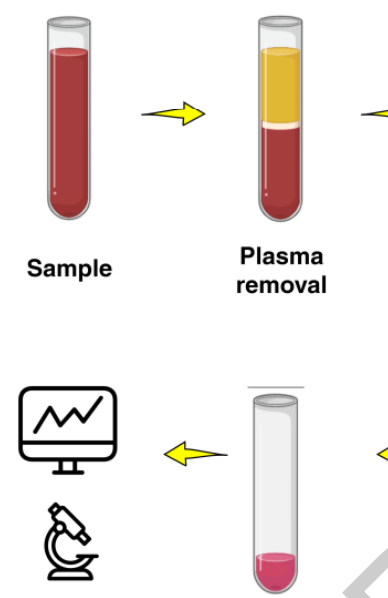

Analysis

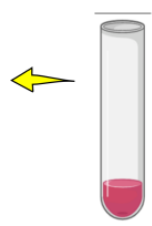

Resuspension

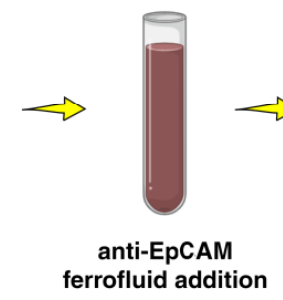

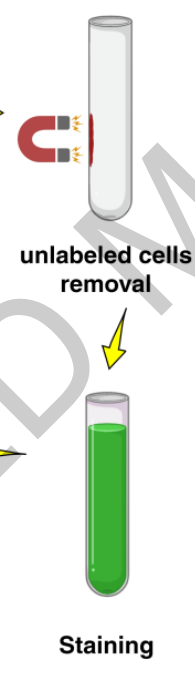

B)

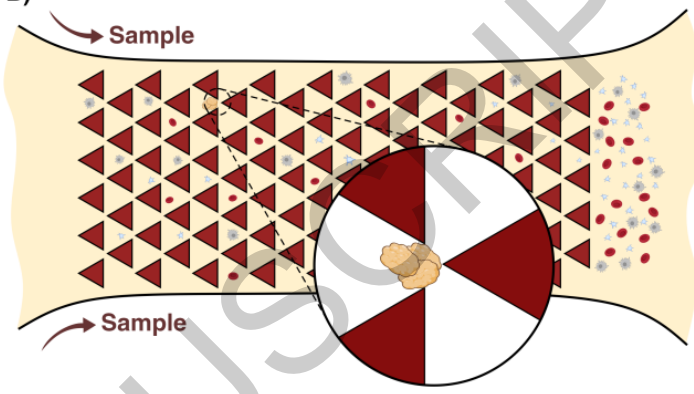

D)

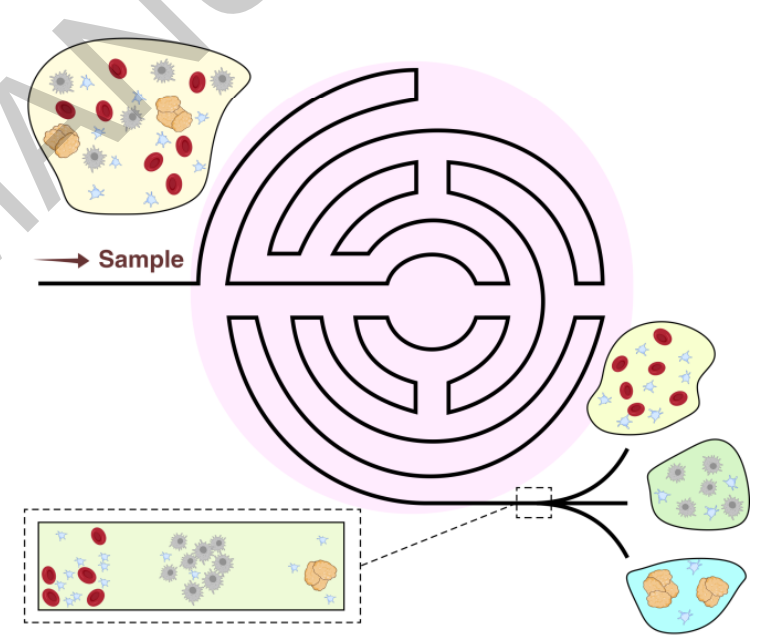

Figure 4. CTC isolation technologies.

(A) Straight microfluidic chip which focuses cells larger than $14 \mu \mathrm{m}$ into the inner channel (including CTC clusters) (B) CTC cluster chip which traps CTC clusters within triangular microposts (C) CellSearch Methodology for capturing EpCAM positive CTCs, including CTC clusters and (D) Labyrinth chip which uses inertial focussing and collection of for CTC and cluster capture. 


\section{Conclusion}

CTC clusters show a vast heterogeneity and their function in metastasis, disease prognosis, patient survival and treatment response are not yet fully ascertained. Therefore, robust characterisation of CTC clusters is needed to improve our understanding of these metastatic precursor cells to develop anti-metastatic treatment strategies. One way the field is rapidly emerging is by advancements in CTC cluster isolation strategies, powered in part by advances in microfluidic technologies. A greater understanding of these cellular aggregates is likely to lead to prognostication tools that can be assessed at time of diagnosis to inform on the likelihood of development of metastasis. More studies are warranted in this field to understand the links between the presence of CTC clusters and the development of metastasis.

\section{Expert Opinion}

Whilst single CTCs have been well documented in the literature, the data on CTC clusters is emerging. The evidence so far supports the notion that CTC clusters are an important population for the process of metastasis. Though the data is emerging, CTC clusters appear to have an increased survival and metastasis forming capacity compared to single CTCs. This may be due to the composition and cooperativity of cells within CTC clusters, including stromal and immune cells, which may provide an immune evasion survival benefit for heterotypic CTC clusters. Interactions of tumour cells and macrophages appear to favour extracellular matrix remodelling, CTC cluster migration and invasiveness. Ultimately, promoting metastasis.

Until recently, it was thought that CTC clusters were unable to traverse through narrow capillaries and that would likely be the point of extravasation. However, their ability to move through narrow vessels was shown as moving in a 'single file' of attached cells, enabled through deformability properties and adhesive properties, with the CTC cluster reforming at the end of the vessel. This explains why tumour-specific tropism of metastasis was not only observed at neighbouring organs but those of distant organs too.

CTC enrichment methodologies have focussed on the capture and characterization of single CTCs using marker-dependent and independent methodologies. In these studies, CTC clusters were only observationally reported. However, with advances in microfluidic technologies, custom CTC cluster capture devices have now been developed. The ability to enrich for CTC 
clusters using these advance methodologies may enable functional characterisation of CTC clusters for "on-chip" drug testing capabilities. The greater characterization of CTC clusters will enable cell-cell interactions to be assessed in greater detail, identifying cell adhesion proteins which may help keep clusters intact. With this knowledge, strategies can be developed to target these cell-cell interactions, to reduce the formation of CTC clusters, and in turn, the formation of CTC-cluster driven metastasis. Reducing CTC clusters into single CTCs may expose the tumour cells to a greater proportion of immune cells.

Clinically, the presence of CTC clusters at the time of diagnosis may indicate patients more likely to develop metastasis, who can be monitored more closely, or treated more aggressively to reduce CTC cluster-mediated metastasis. Ultimately, the presence of CTCs may include that of CTC clusters, which can be tracked over the course of therapy to 1) enumerate CTCs 2) characterise the presence of clinically actionable biomarkers 3) functionally test CTCs ex vivo and 4) identify drug treatment strategies personalised to the patient.

\section{Funding}

This paper was not funded.

\section{Declaration of Interest}

The authors have no relevant affiliations or financial involvement with any organization or entity with a financial interest in or financial conflict with the subject matter or materials discussed in the manuscript. This includes employment, consultancies, honoraria, stock ownership or options, expert testimony, grants or patents received or pending, or royalties.

\section{Reviewer Disclosures}

Peer reviewers on this manuscript have no relevant financial or other relationships to disclose.

\section{Acknowledgements}

A Kulasinghe is supported by an NHMRC ECF fellowship (APP1157741) and Cure Cancer (APP1182179). K O'Byrne is supported by the Princess Alexandra Hospital Foundation grant. 


\section{References}

1. Paget S. The distribution of secondary growths in cancer of the breast. Lancet. 1889:571-573.

2. Massagué J, Obenauf AC. Metastatic colonization by circulating tumour cells. Nature. 2016;529(7586):298-306.

3. Fidler IJ. The relationship of embolic homogeneity, number, size and viability to the incidence of experimental metastasis. European Journal of Cancer (1965). 1973;9(3):223-227.

4. Liotta LA, Kleinerman J, Saldel GM. The significance of hematogenous tumor cell clumps in the metastatic process. Cancer research. 1976;36(3):889-894.

5. Cho $\mathrm{EH}$, Wendel $\mathrm{M}$, Luttgen $\mathrm{M}$, et al. Characterization of circulating tumor cell aggregates identified in patients with epithelial tumors. Physical biology. 2012;9(1):016001.

6. Suo $Y$, Xie $C$, Zhu $X$, et al. Proportion of circulating tumor cell clusters increases during cancer metastasis. Cytometry Part A. 2017;91(3):250-253.

7. Aceto N, Bardia A, Miyamoto DT, et al. Circulating tumor cell clusters are oligoclonal precursors of breast cancer metastasis. Cell. 2014;158(5):1110-1122.

8. Harris TJ, Tepass U. Adherens junctions: from molecules to morphogenesis. Nature reviews Molecular cell biology. 2010;11(7):502-514.

9. Gkountela S, Castro-Giner F, Szczerba BM, et al. Circulating tumor cell clustering shapes DNA methylation to enable metastasis seeding. Cell. 2019;176(1-2):98-112. e14.

10. Yu M, Bardia A, Wittner BS, et al. Circulating breast tumor cells exhibit dynamic changes in epithelial and mesenchymal composition. science. 2013;339(6119):580-584.

11. Aceto N. Bring along your friends: homotypic and heterotypic circulating tumor cell clustering to accelerate metastasis. biomedical journal. 2020;43(1):18-23.

12. Rianna C, Radmacher M, Kumar S. Direct evidence that tumor cells soften when navigating confined spaces. Molecular biology of the cell. 2020:mbc. E19-10-0588.

13. Moro A, Foresta E, Gasparini G, et al. Ameloblastic carcinoma of the maxilla: A case report and an updated review of the literature. Oncology letters. 2016;12(6):4339-4350.

14. Au SH, Storey BD, Moore JC, et al. Clusters of circulating tumor cells traverse capillary-sized vessels. Proceedings of the National Academy of Sciences. 2016;113(18):4947-4952.

15. Hanahan D, Weinberg RA. Hallmarks of cancer: the next generation. cell. 2011;144(5):646-674.

16. Sawabata NJJOTD. Circulating tumor cells in lung cancer: cluster circulating tumor cells as hybrid epithelial-mesenchymal transition/mesenchymal-epithelial transition (E/M). 2017. 2017;9(10):3547-3550.

17. Lee JM, Dedhar S, Kalluri $R$, et al. The epithelial-mesenchymal transition: new insights in signaling, development, and disease. Journal of Cell Biology. 2006;172(7):973-981.

18. Zheng $Q-M$, Chen $X-Y, B a o ~ Q-F$, et al. ILK enhances migration and invasion abilities of human endometrial stromal cells by facilitating the epithelial-mesenchymal transition. Gynecological Endocrinology. 2018;34(12):1091-1096.

19. Van Es JH, Barker N, Clevers H. You Wnt some, you lose some: oncogenes in the Wnt signaling pathway. Current opinion in genetics \& development. 2003;13(1):28-33.

20. Thompson EW, Newgreen DF. Carcinoma invasion and metastasis: a role for epithelialmesenchymal transition? Cancer research. 2005;65(14):5991-5995.

21. Zavadil J, Böttinger EP. TGF- $\beta$ and epithelial-to-mesenchymal transitions. Oncogene. 2005;24(37):5764-5774.

22. Jin H, Varner J. Integrins: roles in cancer development and as treatment targets. British journal of cancer. 2004;90(3):561-565. 
23. Thomas JR, Moore KM, Sproat C, et al. Integrin $\alpha$ V $\beta 6$-EGFR crosstalk regulates bidirectional force transmission and controls breast cancer invasion. bioRxiv. 2018:407908.

24. Mani SA, Guo W, Liao M-J, et al. The epithelial-mesenchymal transition generates cells with properties of stem cells. Cell. 2008;133(4):704-715.

25. Polyak K, Weinberg RA. Transitions between epithelial and mesenchymal states: acquisition of malignant and stem cell traits. Nature Reviews Cancer. 2009;9(4):265-273.

26. Yochum ZA, Cades J, Wang $\mathrm{H}$, et al. Targeting the EMT transcription factor TWIST1 overcomes resistance to EGFR inhibitors in EGFR-mutant non-small-cell lung cancer. Oncogene. 2019;38(5):656-670.

27. Khoo BL, Warkiani ME, Tan DS-W, et al. Clinical validation of an ultra high-throughput spiral microfluidics for the detection and enrichment of viable circulating tumor cells. PloS one. 2014;9(7):e99409.

28. De Craene B, Berx G. Regulatory networks defining EMT during cancer initiation and progression. Nature Reviews Cancer. 2013;13(2):97-110.

29. Vetter M, Landin J, Szczerba BM, et al. Denosumab treatment is associated with the absence of circulating tumor cells in patients with breast cancer. Breast Cancer Research. 2018;20(1):141.

30. Friedlander TW, Premasekharan G, Paris PL. Looking back, to the future of circulating tumor cells. Pharmacology \& therapeutics. 2014;142(3):271-280.

31. Szczerba BM, Castro-Giner F, Vetter M, et al. Neutrophils escort circulating tumour cells to enable cell cycle progression. Nature. 2019;566(7745):553-557.

32. Rostami P, Kashaninejad N, Moshksayan K, et al. Novel approaches in cancer management with circulating tumor cell clusters. Journal of Science: Advanced Materials and Devices. 2019;4(1):118.

33. Sprouse ML, Welte $T$, Boral $D$, et al. PMN-MDSCs enhance CTC metastatic properties through reciprocal interactions via ROS/Notch/Nodal signaling. International journal of molecular sciences. 2019;20(8):1916.

34. Cheung KJ, Padmanaban V, Silvestri V, et al. Polyclonal breast cancer metastases arise from collective dissemination of keratin 14-expressing tumor cell clusters. Proceedings of the National Academy of Sciences. 2016;113(7):E854-E863.

35. Brandt B, Junker R, Griwatz C, et al. Isolation of prostate-derived single cells and cell clusters from human peripheral blood. Cancer research. 1996;56(20):4556-4561.

36. Jansson S, Bendahl P-O, Larsson A-M, et al. Prognostic impact of circulating tumor cell apoptosis and clusters in serial blood samples from patients with metastatic breast cancer in a prospective observational cohort. BMC cancer. 2016;16(1):433.

37. Murlidhar V, Reddy RM, Fouladdel S, et al. Poor prognosis indicated by venous circulating tumor cell clusters in early-stage lung cancers. Cancer research. 2017;77(18):5194-5206.

38. Chistiakov DA, Chekhonin VP. Circulating tumor cells and their advances to promote cancer metastasis and relapse, with focus on glioblastoma multiforme. Experimental and molecular pathology. 2018;105(2):166-174.

39. Mego M, Gao H, Cohen EN, et al. Circulating tumor cells (CTCS) are associated with abnormalities in peripheral blood dendritic cells in patients with inflammatory breast cancer. Oncotarget. 2017 May 30;8(22):35656-35668. doi: 10.18632/oncotarget.10290. PubMed PMID: 27374101; PubMed Central PMCID: PMCPMC5482606. eng.

40. Scheel C, Weinberg RA, editors. Cancer stem cells and epithelial-mesenchymal transition: concepts and molecular links. Seminars in cancer biology; 2012: Elsevier.

41. Kim MS, Sim TS, Kim YJ, et al. SSA-MOA: a novel CTC isolation platform using selective size amplification (SSA) and a multi-obstacle architecture (MOA) filter. Lab on a Chip. 2012;12(16):2874-2880. 
42. Luzzi KJ, MacDonald IC, Schmidt EE, et al. Multistep nature of metastatic inefficiency: dormancy of solitary cells after successful extravasation and limited survival of early micrometastases. The American journal of pathology. 1998;153(3):865-873.

43. Wang C, Mu Z, Chervoneva I, et al. Longitudinally collected CTCs and CTC-clusters and clinical outcomes of metastatic breast cancer. Breast cancer research and treatment. 2017;161(1):8394.

44. Jacot W, Mazel M, Mollevi C, et al. Expression of PD-L1 on circulating breast cancer cells: Correlation with clinicopathologic data and impact on prognosis. AACR; 2018.

45. Kulasinghe A, Perry C, Kenny L, et al. PD-L1 expressing circulating tumour cells in head and neck cancers. BMC cancer. 2017;17(1):333.

46. Brahmer J. PD-1-targeted immunotherapy: recent clinical findings. Clinical advances in hematology \& oncology: H\&O. 2012;10(10):674.

47. Topalian SL, Hodi FS, Brahmer JR, et al. Safety, activity, and immune correlates of anti-PD-1 antibody in cancer. New England Journal of Medicine. 2012;366(26):2443-2454.

48. Mazel M, Jacot W, Pantel K, et al. Frequent expression of PD-L1 on circulating breast cancer cells. Molecular oncology. 2015;9(9):1773-1782.

49. Strati A, Koutsodontis G, Papaxoinis G, et al. Prognostic significance of PD-L1 expression on circulating tumor cells in patients with head and neck squamous cell carcinoma. Annals of Oncology. 2017;28(8):1923-1933.

50. Tian M, Zhang Y, Liu Z, et al. The PD-1/PD-L1 inhibitory pathway is altered in pre-eclampsia and regulates $T$ cell responses in pre-eclamptic rats. Scientific reports. 2016;6(1):1-14.

51. DiDomenico J, Lamano JB, Oyon D, et al. The immune checkpoint protein PD-L1 induces and maintains regulatory T cells in glioblastoma. Oncoimmunology. 2018;7(7):e1448329.

52. Lin A, Yan $W-H$. Human leukocyte antigen-G (HLA-G) expression in cancers: roles in immune evasion, metastasis and target for therapy. Molecular Medicine. 2015;21(1):782-791.

53. Leblanc $R$, Peyruchaud $O$. Metastasis: new functional implications of platelets and megakaryocytes. Blood, The Journal of the American Society of Hematology. 2016;128(1):24-31.

54. Placke $T$, Örgel $M$, Schaller $M$, et al. Platelet-derived MHC class I confers a pseudonormal phenotype to cancer cells that subverts the antitumor reactivity of natural killer immune cells. Cancer Research. 2012;72(2):440-448.

55. Palumbo JS, Talmage KE, Massari JV, et al. Platelets and fibrin (ogen) increase metastatic potential by impeding natural killer cell-mediated elimination of tumor cells. Blood. 2005;105(1):178-185.

56. Gruber I, Landenberger N, Staebler A, et al. Relationship between circulating tumor cells and peripheral T-cells in patients with primary breast cancer. Anticancer Research. 2013;33(5):22332238.

57. Lou X-L, Sun J, Gong S-Q, et al. Interaction between circulating cancer cells and platelets: clinical implication. Chinese Journal of Cancer Research. 2015;27(5):450.

58. Piskounova E, Agathocleous M, Murphy MM, et al. Oxidative stress inhibits distant metastasis by human melanoma cells. Nature. 2015;527(7577):186-191.

59. Alix-Panabières C. EPISPOT assay: detection of viable DTCS/CTCs in solid tumor patients. Minimal Residual Disease and Circulating Tumor Cells in Breast Cancer: Springer; 2012. p. 69-76.

60. $\mathrm{Yu} M$, Stott $S$, Toner $M$, et al. Circulating tumor cells: approaches to isolation and characterization. Journal of Cell Biology. 2011;192(3):373-382.

61. Königsberg R, Obermayr E, Bises $G$, et al. Detection of EpCAM positive and negative circulating tumor cells in metastatic breast cancer patients. Acta oncologica. 2011;50(5):700-710.

62. Kulasinghe A, Zhou J, Kenny L, et al. Capture of circulating tumour cell clusters using straight microfluidic chips. Cancers. 2019;11(1):89. 
63. Pantel K, Alix-Panabières $\mathrm{C}$. Circulating tumour cells in cancer patients: challenges and perspectives. Trends in molecular medicine. 2010;16(9):398-406.

64. Zeinali $M$, Lee $M$, Nadhan $A$, et al. High-throughput label-free isolation of heterogeneous circulating tumor cells and CTC clusters from non-small-cell lung cancer patients. Cancers. 2020;12(1):127.

65. Alix-Panabières C, Pantel K. Challenges in circulating tumour cell research. Nature Reviews Cancer. 2014;14(9):623-631.

66. Poudineh $\mathrm{M}$, Sargent EH, Pantel K, et al. Profiling circulating tumour cells and other biomarkers of invasive cancers. Nature Biomedical Engineering. 2018;2(2):72-84.

67. Alshareef M, Metrakos N, Juarez Perez E, et al. Separation of tumor cells with dielectrophoresisbased microfluidic chip. Biomicrofluidics. 2013;7(1):011803.

68. Tang Y, Shi J, Li S, et al. Microfluidic device with integrated microfilter of conical-shaped holes for high efficiency and high purity capture of circulating tumor cells. Scientific reports. 2014;4:6052.

69. Wan S, Kim TH, Smith KJ, et al. New Labyrinth Microfluidic Device Detects Circulating Tumor Cells Expressing Cancer Stem Cell Marker and Circulating Tumor Microemboli in Hepatocellular Carcinoma. Scientific Reports. 2019 2019/12/09;9(1):18575. doi: 10.1038/s41598-019-54960-y.

70. Fachin F, Spuhler P, Martel-Foley JM, et al. Monolithic chip for high-throughput blood cell depletion to sort rare circulating tumor cells. Scientific reports. 2017;7(1):1-11.

71. Zhou J, Kulasinghe A, Bogseth A, et al. Isolation of circulating tumor cells in non-small-cell-lungcancer patients using a multi-flow microfluidic channel. Microsystems \& nanoengineering. 2019;5(1):1-12.

72. Qian W, Zhang Y, Chen W. Capturing cancer: emerging microfluidic technologies for the capture and characterization of circulating tumor cells. Small. 2015;11(32):3850-3872.

\section{CTC cluster studies}

\begin{tabular}{|c|c|}
\hline Type of cancer & Prognostic significance \\
\hline $\begin{array}{l}\text { Breast, lung and } \\
\text { prostate cancer }\end{array}$ & $\begin{array}{l}\text { CTC clusters derived from its' primary tumour, denoting oligoclonal origin. } \\
\text { Plakoglobin-dependent intercellular adhesions led to strong binding of CTCs and formed C } \\
\text { in breast cancer. } \\
\text { CTC clusters were extremely rare in the circulation and consisted of 2-5\% of all CTCs eve } \\
\text { In lung cancer, CTC clusters were found to be more resistant to apoptosis and had shorter c } \\
\text { clearance rate than single CTCs. } \\
\text { In breast and lung cancers, the presence of CTC clusters showed 23-50 times higher metast } \\
\text { compared to single CTCs. Further, in breast and prostate cancers, CTC-clusters were show } \\
\text { shorter overall survival time and adverse clinical outcomes. }\end{array}$ \\
\hline Breast cancer & $\begin{array}{l}\text { The persistence of apoptotic CTCs or CTC clusters even after initiation of the systemic the } \\
\text { worsen prognosis with lower progression-free survival and overall survival rate than patien } \\
\text { these CTC characteristics in metastatic breast cancer. Thus, in addition to CTC enumeratio } \\
\text { morphological characterization of persistent CTCs was found to be crucial during treatmen }\end{array}$ \\
\hline
\end{tabular}




\begin{tabular}{|c|c|}
\hline $\begin{array}{l}\text { Liver cancer and } \\
\text { the subcutaneous } \\
\text { prostate cancer }\end{array}$ & $\begin{array}{l}\text { Over the cause of liver and prostate cancer metastasis, both CTC clusters and single CTC } \\
\text { elevated while CTC clusters were found to be increased drastically than single CTCs. A } \\
\text { proportion of CTC clusters were found than previously anticipated. Therefore, CTC clus } \\
\text { shown to enhance metastatic potential compared to single CTCs. }\end{array}$ \\
\hline Breast cancer & $\begin{array}{l}\text { CTC-cluster had significantly higher metastasis potential than individual CTCs. } \\
\text { The appearance of larger CTC clusters ( } \geq 5 \text { CTCs) was suggested the higher mortality rat } \\
\text { breast cancer. }\end{array}$ \\
\hline Lung & $\begin{array}{l}\text { The proportion of CTC clusters present in peripheral blood was significantly correlated wi } \\
\text { of lung cancer. } \\
\text { CTC clusters demonstrated the therapeutic resistance, indicating their aggressiveness and } \\
\text { increasing the metastatic ability and ultimately poor prognosis. }\end{array}$ \\
\hline - & $\begin{array}{l}\text { CTC clusters (consisting up to } 20 \text { CTCs) showed the ability to transverse } 5 \text { to } 10 \mu \mathrm{m} \text { capil } \\
\text { vessels by unfolding into single file chains as it reduced the hydrodynamic resistance. Thi } \\
\text { found to be rapid, efficient, reversible and greatly contributed to the dissemination of canc } \\
\text { organs. } \\
\text { Further, preliminary studies revealed that this process could be inhibited by drugs that disn } \\
\text { interactions. }\end{array}$ \\
\hline & $\begin{array}{l}\text { CTC clusters induced a }>15 \text {-fold higher colony forming ability in vitro and }>100 \text {-fold hig } \\
\text { formation ability in vivo. } \\
\text { Expression of the epithelial cytoskeletal protein, Keratin } 14 \text { (K14) in CTC clusters was sig } \\
\text { correlated with higher metastasis rate. } \\
\text { Suppression of K14 exhibition led to lower expression of multiple metastasis effectors inc } \\
\text { Tenascin-C, Jagged-1, Epiregulin and remarkable reduction in metastatic potential. }\end{array}$ \\
\hline & $\begin{array}{l}\text { In breast cancer, CTC-neutrophil clusters denoted the most efficient metastasis seeding ce } \\
\text { and their presence in the bloodstream was associated with a poor prognosis and worsen cl } \\
\text { outcome. } \\
\text { CTCs in CTC-neutrophil clusters showed higher level of positive regulators for cell cycle } \\
\text { replication compared to CTCs alone. } \\
\text { Vascular Cell Adhesion Molecule-1 (VCAM-1) mediated the formation of CTC-neutroph } \\
\text { and inhibition of VCAM-1 prevented the formation of CTC-neutrophil clusters. }\end{array}$ \\
\hline Breast cancer & $\begin{array}{l}\text { In CTC clusters, binding sites for stemness and proliferation associated transcription facto } \\
\text { hypomethylated and transcriptionally activated than single CTCs. } \\
\text { These CTC cluster associated hypomethylated regions were found to be hypomethylated e } \\
\text { primary tumor level in breast cancer patients which had been led to a poor prognosis than }\end{array}$ \\
\hline
\end{tabular}




\begin{tabular}{|l|l}
\hline & $\begin{array}{l}\text { who displayed higher methylation levels in the same regions. } \\
\mathrm{Na}^{+} / \mathrm{K}^{+} \text {ATPase inhibitors and tubulin binding agents were able the disrupt CTC clusters in } \\
\text { cells. It has caused to DNA methylation remodeling at critical sites and suppression of met } \\
\text { potential. }\end{array}$ \\
\hline $\begin{array}{l}\text { BSCLC, } \\
\text { pancreatic cancer } \\
\text { and prostate cancer }\end{array}$ & $\begin{array}{l}\text { The enrichment-free immunofluorescence detection method was a highly sensitive method } \\
\text { enumerate homotypic CTC clusters in patients with breast, NSCLC, pancreatic and prostat } \\
\text { Several differences in physical characteristics of single CTCs and CTC clusters have been } \\
\text { Mean cell length of the single CTCs was two-fold higher than leucocytes, but CTCs in clus } \\
\text { equal in length to the leucocytes. Nuclear to cytoplasmic ratios between single CTCs and C } \\
\text { aggregates were also similar. Together with all the findings, the study has confirmed that C } \\
\text { clusters were smaller than single CTCs. }\end{array}$ \\
\hline $\begin{array}{l}\text { Head and neck } \\
\text { cancers }\end{array}$ & $\begin{array}{l}\text { In head and neck cancers, straight microfluidic chips could be used to capture CTC/CTC cl } \\
\text { and circulating tumour microemboli (CTM) without purification step. This straight microfl } \\
\text { could be used with lower blood dilutions and undiluted whole blood. }\end{array}$ \\
\hline NSCLC & $\begin{array}{l}\text { Microfluidic Labyrinth device was a high throughput, biomarker independent, size bas } \\
\text { technique that used to capture heterogeneous CTCs and CTC clusters in patients with NSC } \\
\text { Heterogeneous CTC populations with either epithelial (EpCAM) or mesenchymal (Viment } \\
\text { markers were able to detect by this technique. As it was a marker independent method, EpC } \\
\text { negative CTCs were also detected efficiently which were often missed by conventional CT } \\
\text { separation methods. } \\
\text { Study further revealed that NSCLC patients who had a higher number of CTC clusters shor } \\
\text { progression free survival than patients with single CTCs. }\end{array}$ \\
\hline
\end{tabular}

